Mitsue Isosaki ${ }^{1}$

Elisabeth Cardoso ${ }^{2}$

Débora Miriam Raab Glina ${ }^{3}$

Anna Carolina Di Creddo Alves ${ }^{4}$

Lys Esther Rocha ${ }^{5}$

\section{Prevalência de sintomas osteomusculares entre trabalhadores de um Serviço de Nutrição Hospitalar em São Paulo, SP*}

\author{
Prevalence of musculoskeletal symptoms among hospital food \\ service workers in São Paulo, Brazil
}

1 Diretor Técnico do Serviço de Nutrição e Dietética do Instituto do Coração do Hospital das Clínicas da Faculdade de Medicina da Universidade de São Paulo, SP, Brasil.

2 Nutricionista chefe do Serviço de Nutrição e Dietética do Instituto do Coração do Hospital das Clínicas da Faculdade de Medicina da Universidade de São Paulo, SP, Brasil.

3 Psicólogo, professor colaborador do Departamento de Medicina Legal, Ética Médica e Medicina Social e do Trabalho da Faculdade de Medicina, Universidade de São Paulo, SP, Brasil.

4 Nutricionista do Serviço de Nutrição e Dietética do Instituto do Coração do Hospital das Clínicas da Faculdade de Medicina da Universidade de São Paulo, SP, Brasil.

5 Médica, Professora Doutora do Departamento de Medicina Legal, Ética Médica e Medicina Social e do Trabalho da Faculdade de Medicina da Universidade de São Paulo, SP, Brasil.

*Artigo baseado na tese de doutorado de Mitsue Isosaki intitulada Intervenção nas situações de trabalho de um serviço de nutrição hospitalar de São Paulo e repercussões nos sintomas osteomusculares, apresentada em 2008 na Faculdade de Medicina da Universidade de São Paulo (USP).

Projeto financiado pela Fapesp: N05/56541-3.

Contato:

Mitsue Isosaki

Avenida Doutor Eneas Carvalho de Aguiar, 44 - Cerqueira Cesar, São Paulo, SP, Brasil

CEP: 05403-000

E-mail:

mitsue.isosaki@incor.usp.br

Recebido: 05/08/2010

Revisado: 21/10/2011

Aprovado: 28/10/2011

\section{Resumo}

Objetivo: identificar a prevalência de sintomas osteomusculares entre trabalhadores de um serviço de nutrição de um hospital público. Método: estudo epidemiológico transversal com aplicação de questionários para 115 trabalhadores (representando 89\% do quadro de pessoal) do serviço de nutrição de um hospital de cardiologia, em São Paulo, em 2007. O instrumento utilizado foi baseado no Questionário Nórdico de Sintomas Osteomusculares de Kuorinka et al. (1987) para identificação de dor ou desconforto relacionados ao trabalho, localização e tipo de queixa, além de caracterizar a frequência, a intensidade e a duração dos sintomas. Resultados: a média da idade dos trabalhadores foi de $37 \pm 9$,8 anos, 81\% eram do sexo feminino, 58\% possuíam Ensino Médio e o tempo médio de trabalho foi de 9,3 \pm 7,5 anos em jornada de trabalho de 40 horas semanais. Do total de participantes, $89 \%$ referiram dor ou desconforto relacionados ao trabalho no último ano em membros inferiores (65\%), ombros (55\%), região lombar (39\%), região cervical (37\%), mãos/punhos/dedos (29\%), coluna (28\%), antebraço (28\%) e cotovelos (10\%). O movimento de andar e transportar carga foi a causa mais citada para os sintomas (31\%). Conclusão: entre os trabalhadores do serviço, a prevalência de sintomas osteomusculares foi alta, principalmente nos membros inferiores e nos ombros. Recomendam-se novas pesquisas que incluam a avaliação do ambiente e das atividades desenvolvidas pelos trabalhadores.

Palavras-chave: serviço hospitalar de nutrição; distúrbio osteomuscular relacionado ao trabalho; LER/DORT; saúde do trabalhador.

\begin{abstract}
Objective: To identify musculoskeletal symptoms among workers from a food service of a public cardiologic hospital located in São Paulo, Brazil. Method: A cross sectional study was carried out in 2007, by applying questionnaires to 115 workers (89\% of total staff). The instrument was based on the Nordic Questionnaire on Musculoskeletal Symptoms by Kuorinka et al. (1987), for identification of any kind of pain or discomfort related to work, its location, type of complaint, and frequency, intensity, and duration of symptoms. Results: The workers' average age was $37 \pm 9.8$ years, $81 \%$ were women, and $58 \%$ had highschool level. They had been working in the hospital for $9.3 \pm 7.5$ years, on a 40-hour week schedule. Most (89\%) of the subjects reported pain or discomfort related to work during the last 12 months, mainly in lower limbs (65\%). Other parts of the body mentioned by the workers were: shoulders (55\%), lumbar region (39\%), cervical region (37\%), wrist/hands/fingers (29\%), spine (28\%), forearm (28\%), and elbows (10\%). The movements made while walking or carrying loads were the most reported cause of symptoms (31\%). Conclusion: The study found a high prevalence of musculoskeletal symptoms among workers from the hospital food service, mainly in lower limbs and shoulders. It is recommended that future studies include assessment of environment and worker's activities.
\end{abstract}

Keywords: hospital food service; work related musculoskeletal disorders; WMSD; occupational health. 


\section{Introdução}

Os serviços de nutrição hospitalar têm por finalidade a prestação de assistência nutricional à comunidade sadia e enferma por meio do fornecimento de refeições, orientação dietoterápica e educação alimentar. No caso de hospitais-escola, atividades de ensino e pesquisa também são realizadas. Esses serviços têm como característica a prestação diária, ininterrupta e contínua do atendimento aos pacientes, e as atividades neles desenvolvidas exigem exatidão, rapidez e sincronia da equipe. Além disso, dependendo da função e do local de trabalho, os seus trabalhadores são submetidos a condições como ruído, calor, umidade, risco de acidentes, esforço físico e/ou mental, ritmo de trabalho intenso, monótono e repetitivo, em posturas estáticas que podem levar à fadiga física, ao estresse e às doenças ocupacionais (SANT'ANA; AZEREDO; CASTRO, 1994; LANCMAN et al., 2000; LEMOS; PROENÇA, 2002; MATOS; PROENÇA, 2003; ISOSAKI, 2003; ISOSAKI, 2008).

Pesquisas sobre Lesões por Esforços Repetitivos (LER)/Distúrbios Osteomusculares Relacionados ao Trabalho (DORT) em serviços de alimentação no Brasil, principalmente na área hospitalar, são poucas: Garcia et al. (1998) estudaram um grupo de 185 trabalhadores da Divisão de Alimentação da Coordenadoria de Assistência Social (Coseas) da Universidade de São Paulo e encontraram, analisando as Comunicações de Acidentes de Trabalho (CAT) emitidas no período de 1 ano, prevalência de $4,3 \%$ de DORT entre todos os trabalhadores da área e de $13 \%$ quando considerado somente o cargo de auxiliar de cozinha. A maior frequência foi entre o gênero feminino e com tempo de início das atividades entre 5 e 10 anos. Segundo os autores, houve provável agravamento das condições de trabalho no período. Casarotto e Mendes (2003), ao comparar a prevalência de doenças ocupacionais, acidentes de trabalho e dores musculoesqueléticas entre trabalhadores de quatro cozinhas industriais e de uma cozinha de hospital pediátrico, observaram maior prevalência de DORT e queixas de dor nos membros inferiores, na região lombar e nos ombros. Encontramos também poucas pesquisas realizadas no exterior sobre o assunto. Chyuan et al. (2004) estudaram 905 trabalhadores de restaurante hoteleiro em Taiwan e observaram a presença de distúrbios osteomusculares em $84 \%$, sendo que $58 \%$ apresentaram sintomas nos ombros. Apesar da alta prevalência, somente $12 \%$ consideraram sua capacidade para o trabalho afetada e 5\% faziam tratamento médico. Paulozzi, Helgerson e Apol (1984) encontraram também, entre trabalhadores de restaurantes e de hotéis de Washington, sintomas consistentes de síndrome do túnel do carpo, principalmente entre as mulheres (27\%) e entre aqueles com mais de 33 anos de idade (29\%), sendo os cozinheiros, independentemente do sexo e da idade, um grupo de risco.

Os fatores de risco associados ao DORT nesses serviços estão relacionados ao ambiente físico (dimensões, configurações e ambiências), aos instrumentos e equipamentos inadequados (dimensões, peso, desenho antiergonômico), à má postura, ao levantamento de pesos acima dos limites recomendados, aos movimentos repetitivos e aos fatores de organização do trabalho (CASAROTTO; MENDES, 2003; SANT'ANA; AZEREDO; CASTRO, 1994; MATOS; PROENÇA, 2003; JORGE et al., 2009).

Em continuidade ao estudo de Isosaki (2003), que encontrou, como causa principal do absenteísmo entre trabalhadores de serviços de nutrição e dietética de dois hospitais em São Paulo, doenças relacionadas às atividades exercidas pelos trabalhadores e, dentre essas, as do sistema osteomuscular e do tecido conjuntivo, propôs-se o presente estudo, com o objetivo de identificar a prevalência desses sintomas por região do corpo e dos fatores contribuintes segundo a opinião dos trabalhadores.

\section{Metodologia}

A pesquisa foi realizada em 2007 em um serviço de nutrição de um hospital público de cardiologia situado em São Paulo (SP) com cerca de 450 leitos, clínicos e cirúrgicos. Após a aprovação prévia pelas Comissões Científica e de Ética do hospital, o projeto foi apresentado para 129 funcionários, em pequenos grupos, em vários horários e de acordo com a disponibilidade nas escalas de trabalho. Nessa ocasião, os trabalhadores foram convidados a participar da pesquisa respondendo a um questionário e, havendo concordância, a assinarem o termo de consentimento livre e esclarecido.

O instrumento foi composto de questionário com variáveis sociodemográficas, história funcional e ocupacional. As questões de múltipla escolha para identificar os sintomas osteomusculares foram baseadas no Questionário Nórdico de Sintomas Osteomusculares criado por Kuorinka et al. (1987), traduzido e validado por Pinheiro e disponível em Ferreira Júnior (2000). É composto de duas partes: a primeira para identificação dos trabalhadores com qualquer tipo de dor ou desconforto relacionados ao trabalho, sua localização e tipo de queixa; e a segunda para caracterizar a frequência, a intensidade e a duração dos sintomas, bem como as consequências para a capacidade de trabalho, acrescidas de questões abertas 
sobre a opinião dos trabalhadores a respeito da causa, dos fatores de piora e de melhora dos sintomas.

Após o pré-teste, os questionários foram aplicados por 7 profissionais externos ao serviço para 115 funcionários (89\% do quadro de pessoal), que concordaram em participar da pesquisa. A entrevista foi individual, previamente agendada, durante a jornada de trabalho e teve a duração de 30 a 60 minutos, de acordo com o grau de compreensão do trabalhador.

Com o uso dos programas Microsoft Excel e SPSS 12.0, a análise dos dados compreendeu um exame descritivo por meio de frequências absolutas e relativas (percentual) das variáveis sociodemográficas, história ocupacional, sintomas osteomusculares e percepção dos sintomas pelos trabalhadores.

\section{Resultados}

A caracterização da população do estudo está descrita na Tabela 1. A população constitui-se, em sua maioria, do sexo feminino com média de idade de $37 \pm 9,8$ anos casados, com filhos e com grau médio de escolaridade. O tempo médio de trabalho dos 115 participantes foi de 9,3 \pm 7,5 anos, em sua maioria na função de atendentes de nutrição (66\%) na cozinha do hospital (46\%) ou nas unidades de internação (51\%), em jornada de trabalho de 40 horas semanais (79\%) (Tabela 2 ).

Em relação aos sintomas osteomusculares relacionados ao trabalho nos últimos 12 meses, as regiões do corpo mais citadas foram: membros inferiores (65\%), ombros (55\%), região lombar (39\%), região cervical (37\%), mãos/punhos/dedos (29\%), coluna (28\%), antebraço (28\%) e cotovelos (10\%).

A maioria dos participantes, dependendo da região do corpo, referiram dores em ambos os lados (45\% a $92 \%$ ), frequentes (69\% a 91\%), com intensidade moderada a forte (88\% a 100\%) e que surgiram há cerca de 3 anos (59\% a 78\%). Essas dores manifestaram-se ao longo dos 30 dias que antecederam a pesquisa, com duração mínima de 3 dias para a maioria dos trabalhadores (56\% a 87\% dependendo da região do corpo). Para $80 \%$ dos participantes, as dores não provocaram necessidade de afastamento do trabalho (Tabela 3).

O movimento de andar e transportar carga durante as atividades diárias foi a causa mais citada para os sintomas (31\%), seguido de postura em pé (16\%). A piora ocorria também com o movimento de deambulação (38\%) e a melhora, somente com o uso de medicamento (39\%) (Tabela 4).

Tabela 1 Características sociodemográficas dos trabalhadores $(n=115)$ de um serviço de nutrição hospitalar, São Paulo, 2007

\begin{tabular}{llcc}
\hline Característica & Categoria & $N$ & $\%$ \\
\hline \multirow{2}{*}{ Sexo } & Feminino & 93 & 81 \\
& Masculino & 22 & 19 \\
& $19 \mid-25$ & & \\
& $25 \mid-35$ & 13 & 11 \\
Faixa etária (anos) & $35 \mid-45$ & 41 & 36 \\
& $45 \mid-55$ & 29 & 25 \\
& $\geq 55$ & 30 & 26 \\
& Fundamental & 2 & 2 \\
Escolaridade & Médio & 67 & 58 \\
& Superior & 10 & 9 \\
& Pós-graduado & 15 & 13 \\
& Solteiro & 43 & 38 \\
& Casado & 58 & 50 \\
& Separado/viúvo & 14 & 12 \\
& & & \\
& Simos & 70 & 61 \\
& Não & 45 & 39 \\
\hline
\end{tabular}


Tabela 2 Caracteríscas do trabalho e das funções exercidas por trabalhadores $(n=115)$ de um serviço de nutrição hospitalar, São Paulo, 2007

\begin{tabular}{|c|c|c|c|}
\hline Característica & Categoria & $N$ & $\%$ \\
\hline \multirow{6}{*}{ Cargo } & Atendente de nutrição & 76 & 66 \\
\hline & Cozinheiro & 10 & 9 \\
\hline & Escriturário + contínuo & 7 & 6 \\
\hline & Técnico em nutrição & 4 & 3 \\
\hline & Nutricionista & 11 & 10 \\
\hline & Chefia & 7 & 6 \\
\hline \multirow{3}{*}{$\begin{array}{l}\text { Área de } \\
\text { trabalho }\end{array}$} & Cozinha & 53 & 46 \\
\hline & Unidades de internação & 59 & 51 \\
\hline & Administração & 3 & 3 \\
\hline \multirow{6}{*}{$\begin{array}{l}\text { Tempo de trabalho no } \\
\text { hospital (anos) }\end{array}$} & $0 \mid-1$ & 9 & 8 \\
\hline & $1 \mid-5$ & 33 & 29 \\
\hline & $5 \mid-10$ & 31 & 27 \\
\hline & $10 \mid-15$ & 16 & 14 \\
\hline & $15 \mid-20$ & 13 & 11 \\
\hline & $20 \mid-30$ & 13 & 11 \\
\hline \multirow{6}{*}{$\begin{array}{l}\text { Tempo na } \\
\text { função (anos) }\end{array}$} & $0 \mid-1$ & 16 & 14 \\
\hline & $1 \mid-5$ & 44 & 38 \\
\hline & $5 \mid-10$ & 24 & 21 \\
\hline & $10 \mid-15$ & 15 & 13 \\
\hline & $15 \mid-20$ & 7 & 6 \\
\hline & $20 \mid-30$ & 9 & 8 \\
\hline \multirow{2}{*}{$\begin{array}{l}\text { Jornada de trabalho } \\
\text { semanal (horas) }\end{array}$} & 40 & 91 & 79 \\
\hline & $\begin{array}{l}\text { Turnos de } 12 \\
\text { por } 36\end{array}$ & 24 & 21 \\
\hline
\end{tabular}

Tabela 3 Características de sintomas osteomusculares relacionados ao trabalho nos últimos 12 meses, por região do corpo, referidos por trabalhadores $(n=102)$ de um serviço de nutrição hospitalar, São Paulo, 2007

\begin{tabular}{|c|c|c|c|c|c|c|c|c|c|c|c|c|c|c|c|c|}
\hline \multirow[b]{2}{*}{ Característica } & \multicolumn{2}{|c|}{ pescoço } & \multicolumn{2}{|c|}{ ombro } & \multicolumn{2}{|c|}{ coluna } & \multicolumn{2}{|c|}{ cotovelo } & \multicolumn{2}{|c|}{ antebraço } & \multicolumn{2}{|c|}{ mãos } & \multicolumn{2}{|c|}{ lombar } & \multicolumn{2}{|c|}{ MMII* } \\
\hline & $N$ & $\%$ & $N$ & $\%$ & $N$ & $\%$ & $N$ & $\%$ & $N$ & $\%$ & $N$ & $\%$ & $N$ & $\%$ & $N$ & $\%$ \\
\hline Presença de sintomas & 43 & 37 & 63 & 55 & 32 & 28 & 12 & 10 & 32 & 28 & 33 & 29 & 46 & 39 & 75 & 65 \\
\hline \multicolumn{17}{|l|}{ Localização } \\
\hline Lado esquerdo & 4 & 9 & 11 & 17 & 2 & 7 & 1 & 8 & 3 & 9 & 3 & 10 & 2 & 4 & 4 & 5 \\
\hline Lado direito & 9 & 21 & 19 & 30 & 2 & 7 & 7 & 59 & 16 & 50 & 15 & 45 & 2 & 4 & 7 & 9 \\
\hline Ambos & 30 & 70 & 33 & 53 & 27 & 86 & 4 & 33 & 13 & 41 & 15 & 45 & 42 & 92 & 64 & 86 \\
\hline
\end{tabular}


Tabela 3 Características de sintomas osteomusculares relacionados ao trabalho nos últimos 12 meses por região do corpo referidos por trabalhadores $(n=102)$ de um serviço de nutrição hospitalar, São Paulo, 2007

\begin{tabular}{|c|c|c|c|c|c|c|c|c|c|c|c|c|c|c|c|c|}
\hline \multirow[b]{2}{*}{ Característica } & \multicolumn{2}{|c|}{ pescoço } & \multicolumn{2}{|c|}{ ombro } & \multicolumn{2}{|c|}{ coluna } & \multicolumn{2}{|c|}{ cotovelo } & \multicolumn{2}{|c|}{ antebraço } & \multicolumn{2}{|c|}{ mãos } & \multicolumn{2}{|c|}{ lombar } & \multicolumn{2}{|c|}{$M M I I^{*}$} \\
\hline & $N$ & $\%$ & $N$ & $\%$ & $N$ & $\%$ & N & $\%$ & $N$ & $\%$ & $N$ & $\%$ & $N$ & $\%$ & $N$ & $\%$ \\
\hline \multicolumn{17}{|c|}{ Surgimento dos sintomas (meses) } \\
\hline $1-12$ & 20 & 47 & 18 & 29 & 14 & 45 & 4 & 33 & 15 & 46 & 14 & 43 & 19 & 41 & 35 & 47 \\
\hline $12-\mid 36$ & 10 & 23 & 23 & 37 & 9 & 29 & 3 & 26 & 9 & 28 & 9 & 27 & 17 & 37 & 21 & 28 \\
\hline $36-60$ & 8 & 19 & 11 & 17 & 3 & 10 & 1 & 8 & 4 & 13 & 2 & 6 & 6 & 13 & 7 & 9 \\
\hline $60-120$ & 3 & 7 & 8 & 13 & 4 & 13 & 4 & 33 & 4 & 13 & 7 & 21 & 3 & 7 & 11 & 15 \\
\hline 120 e + & 2 & 4 & 3 & 6 & 1 & 3 & 0 & 0 & 0 & 0 & 1 & 3 & 1 & 2 & 1 & 1 \\
\hline
\end{tabular}

Frequência dos sintomas

$\begin{array}{lcccccccccccccccc}\text { Sempre } & 12 & 28 & 26 & 41 & 12 & 39 & 7 & 58 & 10 & 31 & 14 & 42 & 14 & 30 & 44 & 59 \\ \text { Com frequência } & 21 & 49 & 28 & 45 & 14 & 45 & 3 & 25 & 12 & 38 & 15 & 46 & 27 & 59 & 24 & 32 \\ \text { Raramente } & 10 & 23 & 9 & 14 & 5 & 16 & 2 & 17 & 10 & 31 & 4 & 12 & 5 & 11 & 7 & 9\end{array}$

Intensidade dos sintomas

$\begin{array}{lcccccccccccccccc}\text { Forte } & 21 & 49 & 39 & 62 & 16 & 51 & 8 & 67 & 12 & 38 & 14 & 42 & 27 & 59 & 48 & 67 \\ \text { Moderado } & 20 & 46 & 23 & 36 & 13 & 42 & 4 & 33 & 16 & 50 & 18 & 55 & 16 & 35 & 25 & 33 \\ \text { Leve } & 2 & 5 & 1 & 2 & 2 & 7 & 0 & 0 & 4 & 12 & 1 & 3 & 3 & 6 & 2 & 3\end{array}$

Manifestação nos últimos 30 dias

\begin{tabular}{|c|c|c|c|c|c|c|c|c|c|c|c|c|c|c|c|}
\hline Sim & 29 & 67 & 43 & 69 & 23 & 74 & 10 & 83 & 18 & 56 & 27 & 82 & 32 & 70 & 58 \\
\hline Não & 14 & 33 & 20 & 31 & 8 & 26 & 2 & 17 & 14 & 44 & 6 & 18 & 14 & 30 & 17 \\
\hline
\end{tabular}

Manifestação nos últimos 7 dias

$\begin{array}{lcccccccccccccccc}\text { Sim } & 30 & 70 & 46 & 73 & 23 & 74 & 10 & 83 & 19 & 60 & 24 & 73 & 33 & 72 & 65 & 87 \\ \text { Não } & 13 & 30 & 17 & 27 & 8 & 26 & 2 & 17 & 13 & 40 & 9 & 27 & 13 & 28 & 10 & 13\end{array}$

Faltas no trabalho no último ano (dias)

\begin{tabular}{lcccccccccccccccc} 
Nenhum & 34 & 79 & 54 & 86 & 27 & 87 & 9 & 76 & 27 & 85 & 30 & 91 & 37 & 80 & 61 & 81 \\
1 a 7 & 5 & 12 & 6 & 9 & 2 & 7 & 1 & 8 & 2 & 6 & 2 & 6 & 5 & 11 & 9 & 12 \\
8 a 14 & 3 & 7 & 2 & 3 & 1 & 3 & 1 & 8 & 2 & 6 & 1 & 3 & 3 & 7 & 2 & 2 \\
15 e + & 1 & 2 & 1 & 2 & 1 & 3 & 1 & 8 & 1 & 3 & 0 & 0 & 1 & 2 & 3 & 7 \\
\hline
\end{tabular}

* MMII = membros inferiores 
Tabela 4 Opiniões dos trabalhadores de um serviço de nutrição hospitalar sobre a causa e os fatores de piora e melhora dos sintomas osteomusculares relacionados à atividade do trabalho, São Paulo, 2007

\begin{tabular}{|c|c|c|c|}
\hline Fatores & & $N^{*}$ & $\%$ \\
\hline \multirow{9}{*}{ Causa } & deslocamento no trabalho & 61 & 31 \\
\hline & postura & 32 & 16 \\
\hline & $\begin{array}{l}\text { levantamento/transporte/descarga } \\
\text { de material }\end{array}$ & 31 & 15 \\
\hline & organização do trabalho & 20 & 10 \\
\hline & condições ambientais & 14 & 7 \\
\hline & equipamento/mobiliário & 13 & 7 \\
\hline & tensão emocional & 10 & 5 \\
\hline & tarefa & 9 & 5 \\
\hline & outros & 9 & 4 \\
\hline \multirow{10}{*}{ Piora } & deslocamento no trabalho & 52 & 38 \\
\hline & tarefa & 35 & 25 \\
\hline & $\begin{array}{l}\text { levantamento/transporte/descarga } \\
\text { de material }\end{array}$ & 14 & 10 \\
\hline & organização do trabalho & 13 & 9 \\
\hline & postura & 12 & 9 \\
\hline & equipamento/mobiliário & 5 & 4 \\
\hline & tensão emocional & 2 & 1 \\
\hline & relacionamento no trabalho & 2 & 1 \\
\hline & condições ambientais & 1 & 1 \\
\hline & outros & 3 & 2 \\
\hline \multirow{8}{*}{ Melhora } & medicamento & 60 & 39 \\
\hline & repouso & 32 & 21 \\
\hline & fisioterapia & 21 & 13 \\
\hline & alongamento & 16 & 10 \\
\hline & alteração de postura/pausa & 15 & 10 \\
\hline & uso de meia elástica & 6 & 4 \\
\hline & melhoria no trabalho & 3 & 2 \\
\hline & outro & 2 & 1 \\
\hline
\end{tabular}

" Os participantes assinalaram mais de uma resposta.

\section{Discussão}

Na literatura, é bem fundamentada a caracterização de LER/DORT como fenômeno resultante da superutilização das estruturas anatômicas do sistema osteomuscular e da falta de tempo para sua recuperação. Como fatores de risco, destacam-se: a repetitividade de movimentos, a manutenção de posturas inadequadas por tempo prolongado, o esforço físico, o trabalho muscular estático, a pressão mecânica sobre determinados segmentos do corpo, frio, vibração, invariabilidade e conteúdo pobre das tarefas, ritmo intenso de trabalho e fatores organizacionais e psicossociais (BONGERS et al., 1993; PUSTIGLIONE, 1997; ROCHA; FERREIRA JUNIOR, 2000; JORGE et al., 2009; PEHKONEN et al., 2009).

Os serviços de nutrição hospitalar caracterizam-se por terem uma população majoritariamente do sexo feminino, com escolaridade até o Ensino Médio, com filhos (MEZOMO, 1985; ISOSAKI, 2003) e apresentando vários fatores de risco relacionados ao ambiente (ruído, calor, espaço físico), ao posto 
(postura do trabalhador e método de trabalho) e à organização do trabalho (carga e ritmo de trabalho, movimentos repetitivos), além dos fatores psicossociais que podem ser associados às LER/DORT entre os seus trabalhadores (PROENÇA; MATOS, 1996; LIMA et al., 1998; MATOS; PROENÇA, 2003; ISOSAKI, 2008; JORGE et al., 2008).

No presente estudo, $89 \%$ dos trabalhadores referiram dor ou desconforto nos últimos 12 meses, dado semelhante ao encontrado por Chyuan et al. (2004), que observaram a presença de distúrbios osteomusculares em $84 \%$ dos trabalhadores de um restaurante hoteleiro. No mesmo estudo, 58\% apresentaram sintomas nos ombros, valor similar ao encontrado no presente trabalho, que foi de 55\%. Alves (1995), em estudo realizado em um restaurante universitário, observou que $88 \%$ dos trabalhadores referiram problemas nos membros superiores relacionados ao trabalho. Em nosso estudo, as altas prevalências podem estar associadas às posturas adotadas pelos trabalhadores no abastecimento dos alimentos em equipamentos de grande porte (caldeiras), no uso de espátulas de tamanho pequeno para misturar alimentos preparados em chapa, por movimentos repetitivos, como na lavagem das louças, e por fatores da organização do trabalho, como volume de trabalho elevado e pressão temporal em função dos horários das refeições. Estas mesmas condições foram descritas por Masculo et al. (1997), que realizaram estudo de caso em cozinha hospitalar na Paraíba.

A alta prevalência de sintomas osteomusculares nos membros inferiores (65\%) referidos pelos participantes de nosso estudo justifica-se pela atividade exercida em postura em pé, parada ou deambulando, por cerca de $70 \%$ dos trabalhadores em jornadas diárias prolongadas de trabalho (de 8 a 12 horas diárias) sem a realização das pausas recomendadas e constantes nos roteiros de trabalho determinados pelo serviço. Ramazzini (1971), em seu livro publicado em 1700, já descrevia a relação entre a postura em pé e doenças dos membros inferiores. Além da postura, as condições ambientais como a temperatura e umidade relativa do ar elevados, o carregamento de peso, o volume de trabalho, monótono e repetitivo e em ritmo intenso, são fatores que contribuem para o desencadeamento ou o agravamento de transtornos circulatórios nos membros inferiores, como edema e doença venosa, conforme observado por Bertoldi e Proença (2008) em seu estudo.

As dores nos membros superiores e na região do pescoço incidem também em trabalhadores de empresas de processamento de alimentos, conforme estudos de Moore e Garg (1994), Ohlsson, Hansson e Balogh (1994) e Osorio et al. (1994). No estudo de Alves (1995), constatou-se que os trabalhadores com problemas nos membros superiores apresentavam queixas nas seguintes regiões do corpo: pescoço (43,3\%), ombro $(76,7 \%)$, antebraço (60\%), cotovelo (23,3\%), braço $(76,7 \%)$, punho (60\%), mão (50\%), dedos (36,7\%). Em nosso estudo, foram observadas prevalências de $29 \%$ nas mãos/ punhos, 28\% nos antebraços, 10\% nos cotovelos e 37\% no pescoço. As tarefas exercidas em postura estática e em movimentos repetitivos, como no pré-preparo (corte de carnes), na cocção de alimentos e na distribuição de bandejas em esteira rolante, provavelmente seriam os fatores contribuintes.

As principais causas mencionadas pelos participantes para os sintomas osteomusculates foram os movimentos de empurrar carros para transporte de refeições e o levantamento, o transporte e a descarga de gêneros alimentícios e de materiais. No trabalho realizado em um restaurante universitário, Alves (1995) observou que as características do trabalho que contribuíram para a ocorrência dos sintomas estavam relacionadas ao levantamento e ao suporte de peso, aos movimentos repetitivos e ao trabalho em pé e com poucas possibilidades de variação da postura.

O aparecimento dos sintomas nos últimos 12 meses que antecederam a pesquisa, independentemente da região do corpo, provavelmente ocorreu devido ao aumento do número de leitos no hospital, com consequente aumento da carga de trabalho sem a respectiva contratação de funcionários e sem a melhora das condições de trabalho. A situação é preocupante, pois muitos trabalhadores manifestaram dores com duração mínima de 3 dias nos últimos 30 dias.

A despeito das dores, a maioria não se afastou do trabalho, o que sugere a utilização de estratégias como a automedicação e a procura de assistência médica somente em situações mais graves. Segundo os próprios trabalhadores, o uso de medicamentos é o que ocasiona a melhora dos sintomas.

A compreensão dos resultados não é simples, uma vez que os distúrbios osteomusculares relacionados ao trabalho têm origem multicausal. Fatores de organização do trabalho, como a não reposição de funcionários aposentados ou em licenças médicas de longa duração, bem como a não substituição dos trabalhadores que, por "restrições médicas”, não podem desempenhar todas as atividades do cargo para o qual foram contratados, geram sobrecarga de trabalho aos funcionários assíduos e podem estar contribuindo para a alta prevalência dos sintomas osteomusculares entre os trabalhadores do serviço estudado. De acordo com a análise de dados obtidos nos relatórios anuais do serviço de nutrição e da instituição onde foi realizado o estudo, nos últimos 25 anos, houve aumento no número de leitos de 32 para 450, com consequente elevação das internações e da produção de refeições, que passou de 105.000 pra 1.005.000 ao ano, representando um aumento de 
1.000\% sem o respectivo investimento na área da cozinha. Além disso, apesar das inovações introduzidas no serviço nos anos 1990, como a aquisição de fornos combinados, de bandejas térmicas e de alimentos (carnes, vegetais) pré-processados, que trouxeram agilidade e simplificação nos processos de produção, a não ampliação da cozinha e o não redimensionamento proporcional do quadro de funcionários podem também estar repercutido fortemente na saúde dos trabalhadores.

O desenho de corte transversal deste estudo não permite inferência acerca da relação causa-efeito, entretanto, fornece dados específicos importantes sobre os trabalhadores de serviços de nutrição hospitalar que, embora prioritários, são pouco estudados e valorizados no que se refere à promoção da saúde de seus trabalhadores.

\section{Conclusão}

Embora as influências de fatores sociodemográficas, como idade, sexo e atividades domésticas, não possam ser descartadas, os sintomas osteomusculares, principalmente nos membros inferiores referidos na população estudada, possivelmente estão relacionados às atividades dos trabalhadores que passam a maior parte da jornada em pé, parados ou andando, transportando cargas (gêneros alimentícios e carros para transporte de refeições destinadas aos pacientes internados).

Este estudo traz contribuições importantes ao tema devido à carência de pesquisas nesta área. Recomenda-se que o mesmo seja complementado com avaliações do ambiente e das atividades de trabalho realizadas por especialistas.

\section{Contribuição de autoria}

Isosaki, M.: contribuiu no planejamento e no delineamento do projeto, na análise e na interpretação dos dados, na elaboração e na revisão crítica do manuscrito e na aprovação final da versão a ser publicada. Cardoso, E.: contribuiu na análise e na interpretação dos dados, na elaboração e na revisão crítica do manuscrito e na aprovação final da versão a ser publicada. Glina, D. M. R.: contribuiu na análise e na interpretação dos dados e na elaboração do manuscrito. Alves, A. C. Di C.: contribuiu na coleta, na análise e interpretação dos dados e na elaboração do manuscrito. Rocha, L. E.: contribuiu no planejamento, no delineamento e na coordenação do projeto, na análise e na interpretação dos dados, na elaboração e na revisão crítica do manuscrito e na aprovação final da versão a ser publicada.

\section{Referências}

ALVES, G. B. O. Contribuições da ergonomia ao estudo da LER em trabalhadores de um restaurante universitário. 1995. Dissertação (Mestrado em Engenharia de Produção) Disponível em: <http://www. eps.ufsc.br/disserta/gisele/indice/>. Acesso em: 29 nov. 2011.

BERTOLDI, C. M. da L.; PROENÇA, R. P. da C. Doença venosa e sua relação com as condições de trabalho no setor de produção de refeições. Revista de Nutrição, Campinas, v. 21, n. 4, p. 447-454, 2008.

BONGERS, P. M. et al. Psychosocial factors at work and musculoskeletal disease. Scandinavian Journal of Work, Environment \& Health, Finland, v. 19, n. 5, p. 297-312, 1993.

CASAROTTO, R. A.; MENDES, L. F. Queixas, doenças ocupacionais e acidentes de trabalho em trabalhadores de cozinhas industriais. Revista Brasileira de Saúde Ocupacional, São Paulo, v. 28, n. 107/108, p. 109-126, 2003.

CHYUAN, J. Y. et al. Musculoskeletal disorders in hotel restaurant workers. Occupational Medicine, v. 54, n. 1, p. 55-7, 2004.
FERREIRA JUNIOR, M. Saúde no trabalho: temas básicos para o profissional que cuida da saúde dos trabalhadores. São Paulo: Roca, 2000.

GARCIA, M. E. et al. Distúrbios ósteo-musculares relacionados ao trabalho na Coordenadoria de Assistência Social da Universidade de São Paulo. Revista de Medicina do HU-USP, São Paulo, v. 8, n. 1, p. 21-4, 1998.

ISOSAKI, M. Absenteísmo entre trabalhadores de Serviços de Nutrição e Dietética de dois hospitais em São Paulo. Revista Brasileira de Saúde Ocupacional, São Paulo, v. 28, n. 107/108, p. 107-118, 2003.

. Intervenção nas situações de trabalho em um serviço de nutrição hospitalar de São Paulo e repercussões nos sintomas osteomusculares. Saúde, Ética \& Justiça, São Paulo, v. 13, n. 2, p.106-108, 2008.

JORGE, A. T. et al. Fatores de risco associados à satisfação no trabalho em trabalhadores de nutrição hospitalar. Saúde, Ética \& Justiça, São Paulo, v. 13, p. 95-103, 2008.

JORGE, A. T. et al. Distúrbios osteomusculares do trabalho: fatores de risco em trabalhadores de nutrição 
hospitalar. Revista Brasileira de Medicina do Trabalho, São Paulo, v. 7, p. 1-10, 2009.

KUORINKA, I. et al. Standardized Nordic questionnaire for the analysis of musculoskeletal symptoms. Applied Ergonomics, v. 18, n. 3, p. 233-237, 1987.

LANCMAN, S. et al. Estudo e intervenção no processo de trabalho em um restaurante universitário - em busca de novas metodologias. Revista de Terapia Ocupacional da Universidade de São Paulo, v. 11, n. 2/3, p. 79-89, 2000.

LEMOS, M. P.; PROENÇA, R. P. C. Contribuições da ergonomia na melhoria da qualidade higiênicosanitária de refeições coletivas: um estudo de caso. Higiene Alimentar, v. 16, p. 29-34, 2002.

LIMA, F. P. A. et al. O. A produção das lesões por esforços repetitivos num restaurante universitário: análise ergonômica e psicossocial. In: LIMA, M. E. A.; et al. L.E.R: lesões por esforços repetitivos - dimensões ergonômicas e psicossociais. Belo Horizonte: Livraria e Editora Saúde, 1998. p. 108-177.

MASCULO, F. S. et al. O serviço de nutrição e dietética hospitalar - um estudo de caso sob enfoque ergonômico. In: ENCONTRO NACIONAL DE ENGENHARIA DE PRODUÇÃO, 17., e INTERNATIONAL CONGRESS IN INDUSTRIAL ENGINEERING, 3., 1997, Gramado-RS. Anais... Porto Alegre: UFGRS, 1997. v. 1. p. 99-109.

MATOS, C. H.; PROENÇA, R. P. C. Condições de trabalho e estado nutricional de operadores do setor de alimentação coletiva: um estudo de caso. Revista de Nutrição, Campinas, v. 16, n. 4, p. 493-502, 2003.

MEZOMO, I. F. B. O serviço de nutrição: administração e organização. São Paulo: Cedas, 1985.

MOORE, J. S.; GARG, A. Upper extremity disorders in a pork processing plant: relationships between job risk factors and morbidity. American Industrial Hygiene Association Journal, v. 55, n. 8, p. 703-715, 1994.
OHLSSON, K.; HANSSON, G. A.; BALOGH, I. Disorders of the neck and upper in women in a fish processing industry. Occupational and Environmental Medicine, v. 51, n. 12, p. 826-832, 1994.

OSORIO, A. M. et al. Carpal tunnel syndrome among grocery store workers. American Journal of Industrial Medicine, v. 25, n. 2, p. 229-45, 1994.

PAULOZZI, L.; HELGERSON, S. D.; APOL, A. Symptoms consistent with carpal tunnel syndrome among hotel and restaurant workers. Journal of Occupational Medicine, v. 26, n. 9, p. 634, 1984.

PEHKONEN, I. et al. Prospective study on shoulder symptoms among kitchen workers in relation to selfperceived and observed work load. Occupational and Environmental Medicine, v. 66, p. 416-423, 2009.

PROENÇA, R. P. C.; MATOS, C. H. Condições de trabalho e saúde na produção de refeições em creches municipais de Florianópolis. Revista de Ciências da Saúde, v. 15, n. 1/2, p. 73-79, 1996.

PUSTIGLIONE, M. L.E.R. (Lesões por esforço repetitivo): um modelo não reducionista de interpretação. O Mundo da Saúde, v. 21, n. 5, p. 280284, 1997.

RAMAZZINI, B. As doenças dos trabalhadores. Tradução Raimundo Estrela. Rio de Janeiro: Liga Brasileira Contra Acidentes de Trabalho, 1971.

ROCHA, L. E.; FERREIRA JUNIOR, M. Distúrbios osteomusculares relacionados ao trabalho (DORT). In: FERREIRA JUNIOR, M. Saúde no trabalho: temas básicos para o profissional que cuida da saúde dos trabalhadores. São Paulo: Roca, 2000. p. 286-319.

SANT'ANA, H. M. P.; AZEREDO, R. M. C.; CASTRO, J. R. Estudo ergonômico em serviços de alimentação. Saúde em debate, v. 42, p. 45-48, 1994. 